\title{
CLASSIFICATION OF CUNTZ-KRIEGER ALGEBRAS BY ORBIT EQUIVALENCE OF TOPOLOGICAL MARKOV SHIFTS
}

\author{
KENGO MATSUMOTO \\ (Communicated by Bryna Kra)
}

\begin{abstract}
Let $A, B$ be square irreducible matrices with entries in $\{0,1\}$. Assume that the determinants of $1-A$ and $1-B$ have the same sign. We will show that the Cuntz-Krieger algebras $\mathcal{O}_{A}$ and $\mathcal{O}_{B}$ are isomorphic if and only if the right one-sided topological Markov shifts $\left(X_{A}, \sigma_{A}\right)$ and $\left(X_{B}, \sigma_{B}\right)$ are continuously orbit equivalent.
\end{abstract}

\section{INTRODUCTION}

Topological Markov shifts are the basic building blocks of symbolic dynamical systems. Their dynamical properties are closely related to the algebraic properties of the Cuntz-Krieger algebras. There are many interesting approaches to study classification of Cuntz-Krieger algebras from the viewpoints of dynamical properties of the topological Markov shifts ([6], 9, [11, 15], 16, 21, 28, etc.). In particular it has been clarified that there is an interesting relationship between the flow equivalence relation of the topological Markov shifts and the stable isomorphism relation of the Cuntz-Krieger algebras ([6, [9, [1], 15, [16]). In other topological dynamical systems, Giordano-Putnam-Skau [12, [13] (cf. 114, 26], etc.) have proved that two Cantor minimal systems are strongly orbit equivalent if and only if the associated $C^{*}$-crossed products are isomorphic. J. Tomiyama [29] (cf. [4, [30]) has studied relationships between orbit equivalence and $C^{*}$-crossed products for topological free homeomorphisms on compact Hausdorff spaces. The class of one-sided topological Markov shifts is an important class of topological dynamical systems on Cantor sets with continuous surjections that are not homeomorphisms. In a recent paper [21], the author has shown that the one-sided topological Markov shifts $\left(X_{A}, \sigma_{A}\right)$ and $\left(X_{B}, \sigma_{B}\right)$ for irreducible matrices $A$ and $B$ with entries in $\{0,1\}$ satisfying condition (I) 1 are continuously orbit equivalent if and only if there exists an isomorphism between the Cuntz-Krieger algebras $\mathcal{O}_{A}$ and $\mathcal{O}_{B}$ preserving their canonical commutative $C^{*}$-subalgebras $\mathfrak{D}_{A}\left(=C\left(X_{A}\right)\right)$ and $\mathfrak{D}_{B}\left(=C\left(X_{B}\right)\right)$. Keeping in mind the above Giordano-Putnam-Skau works, we would expect that the isomorphism class of the $C^{*}$-algebras completely determines an orbit equivalence class of the underlying topological dynamical systems. The isomorphism class of

Received by the editors July 13, 2011 and, in revised form, October 12, 2011.

2010 Mathematics Subject Classification. Primary 46L55; Secondary 46L35, 37B10.

Key words and phrases. Cuntz-Krieger algebras, topological Markov shifts, orbit equivalence, $K$-theory, flow equivalence.

${ }^{1}$ A matrix $A$ with entries in $\{0,1\}$ is said to satisfy condition (I) if the shift space $X_{A}$ is homeomorphic to a Cantor discontinuum (see [9]). 
the Cuntz-Krieger algebras has been completely classified by its $K$-theory data in Rørdam's paper 28. In this paper, we will prove the following theorems.

Theorem 1.1 (Theorem 4.1). Let $A$ and $B$ be two irreducible square matrices with entries in $\{0,1\}$ satisfying condition (I). If there exists an isomorphism $\alpha$ : $K_{0}\left(\mathcal{O}_{A}\right) \longrightarrow K_{0}\left(\mathcal{O}_{B}\right)$ such that $\alpha\left(\left[1_{A}\right]\right)=\left[1_{B}\right]$ and $\operatorname{det}(1-A)=\operatorname{det}(1-B)$, then there exists an isomorphism $\Psi: \mathcal{O}_{A} \rightarrow \mathcal{O}_{B}$ such that $\Psi\left(\mathfrak{D}_{A}\right)=\mathfrak{D}_{B}$ and $\Psi_{*}=\alpha$, where $1_{A}$ and $1_{B}$ denote the units of $\mathcal{O}_{A}$ and of $\mathcal{O}_{B}$ respectively.

By using the results in [21] and 28], we have

Theorem 1.2 (Theorem 4.3). Let $A$ and $B$ be two irreducible square matrices with entries in $\{0,1\}$ satisfying condition (I). Suppose that the determinants of $1-A$ and $1-B$ have the same sign (or at least one of the determinants is zero). Then the Cuntz-Krieger algebras $\mathcal{O}_{A}$ and $\mathcal{O}_{B}$ are isomorphic if and only if the one-sided topological Markov shifts $\left(X_{A}, \sigma_{A}\right)$ and $\left(X_{B}, \sigma_{B}\right)$ are continuously orbit equivalent.

Under the assumption that the determinants of $1-A$ and $1-B$ have the same sign, the isomorphism classes of the Cuntz-Krieger algebras are completely classified by the continuous orbit equivalence classes of the underlying one-sided topological Markov shifts. The proof for Theorem 1.1 is given by an analogous method to the proof of [28, Theorem 6.5$]$ and some $K$-theoretic ideas.

If the sizes of the matrices are less than or equal to three, the author in 22 . has shown that the isomorphism classes of the Cuntz-Krieger algebras bijectively correspond to the continuous orbit equivalence classes of the underlying one-sided topological Markov shifts without assuming the determinant conditions. Its proof is due to a classification result by Enomoto-Fujii-Watatani [10] of the Cuntz-Krieger algebras whose sizes of the matrices are three. They have used a graph-theoretical technique. By applying Theorem 1.2, one may directly see the result shown in 22 ] (Theorem 4.5).

D. Huang in [15] has proved that any automorphism of the Bowen-Franks group $B F(A)$ (which is isomorphic to $K_{0}\left(\mathcal{O}_{A}\right)$ ) comes from a flow equivalence of the twosided topological Markov shift $\left(\bar{X}_{A}, \bar{\sigma}_{A}\right)$. Analogously to Huang's result, we will see that any automorphism of the $K_{0}$-group $K_{0}\left(\mathcal{O}_{A}\right)$ keeping the class $\left[1_{A}\right]$ of the unit $1_{A}$ of $\mathcal{O}_{A}$ comes from a continuous orbit equivalence of the one-sided topological Markov shift $\left(X_{A}, \sigma_{A}\right)$ (Proposition 5.1). As a corollary for Theorem 1.1 we see

Corollary 1.3. Assume that $A$ is an irreducible square matrix with entries in $\{0,1\}$ satisfying condition (I). For any automorphism $\alpha$ on the Cuntz-Krieger algebra $\mathcal{O}_{A}$, there exists an automorphism $\alpha_{h}$ on $\mathcal{O}_{A}$ induced from a homeomorphism $h$ which gives rise to a continuous orbit equivalence of the one-sided topological Markov shift $\left(X_{A}, \sigma_{A}\right)$ such that $\alpha_{h}\left(\mathfrak{D}_{A}\right)=\mathfrak{D}_{A}$ and $\alpha_{h *}=\alpha_{*}$ on $K_{0}\left(\mathcal{O}_{A}\right)$.

\section{Preliminaries}

Let $A=[A(i, j)]_{i, j=1}^{N}$ be an $N \times N$ matrix with entries in $\{0,1\}$, where $1<N \in$ $\mathbb{N}$. Throughout the paper, we assume that $A$ has no rows or columns identically equal to zero. We denote by $X_{A}$ the shift space

$$
X_{A}=\left\{\left(x_{n}\right)_{n \in \mathbb{N}} \in\{1, \ldots, N\}^{\mathbb{N}} \mid A\left(x_{n}, x_{n+1}\right)=1 \text { for all } n \in \mathbb{N}\right\}
$$

of the right one-sided topological Markov shift for $A$. It is a compact Hausdorff space in the natural product topology on $\{1, \ldots, N\}^{\mathbb{N}}$. The shift transformation 
$\sigma_{A}$ on $X_{A}$ defined by $\sigma_{A}\left(\left(x_{n}\right)_{n \in \mathbb{N}}\right)=\left(x_{n+1}\right)_{n \in \mathbb{N}}$ is a continuous surjective map on $X_{A}$. The topological dynamical system $\left(X_{A}, \sigma_{A}\right)$ is called the (right) one-sided topological Markov shift for $A$. We henceforth assume that $A$ satisfies condition (I) in the sense of Cuntz-Krieger 9 .

A word $\mu=\mu_{1} \cdots \mu_{k}$ for $\mu_{i} \in\{1, \ldots, N\}$ is said to be admissible for $X_{A}$ if $\mu$ appears somewhere in some element $x$ in $X_{A}$. The length of $\mu$ is $k$ and denoted by $|\mu|$. We denote by $B_{k}\left(X_{A}\right)$ the set of all admissible words of length $k$. We set $B_{*}\left(X_{A}\right)=\bigcup_{k=0}^{\infty} B_{k}\left(X_{A}\right)$, where $B_{0}\left(X_{A}\right)$ denotes the empty word $\emptyset$. Denote by $U_{\mu}$ the cylinder set $\left\{\left(x_{n}\right)_{n \in \mathbb{N}} \in X_{A} \mid x_{1}=\mu_{1}, \ldots, x_{k}=\mu_{k}\right\}$ for $\mu=\mu_{1} \cdots \mu_{k} \in$ $B_{k}\left(X_{A}\right)$. The clopen sets $U_{\mu}, \mu \in B_{*}\left(X_{A}\right)$ form an open basis of the topology of $X_{A}$.

The Cuntz-Krieger algebra $\mathcal{O}_{A}$ for the matrix $A$ has been defined in [9] as the universal $C^{*}$-algebra generated by $N$ partial isometries $S_{1}, \ldots, S_{N}$ satisfying the relations

$$
\sum_{j=1}^{N} S_{j} S_{j}^{*}=1, \quad S_{i}^{*} S_{i}=\sum_{j=1}^{N} A(i, j) S_{j} S_{j}^{*}, \quad i=1, \ldots, N
$$

The algebra $\mathcal{O}_{A}$ is the unique $C^{*}$-algebra subject to the relations (2.1) under condition (I) for $A$. If in particular $A$ is irreducible, it is a simple $C^{*}$-algebra ([9]). For a word $\mu=\mu_{1} \cdots \mu_{k}$ with $\mu_{i} \in\{1, \ldots, N\}$, we denote $S_{\mu_{1}} \cdots S_{\mu_{k}}$ by $S_{\mu}$. Then $S_{\mu} \neq 0$ if and only if $\mu \in B_{*}\left(X_{A}\right)$. Let $\mathfrak{D}_{A}$ be the $C^{*}$-subalgebra of $\mathcal{O}_{A}$ generated by the projections of the form $S_{\mu} S_{\mu}^{*}, \mu \in B_{*}\left(X_{A}\right)$. It is isomorphic to the commutative $C^{*}$-algebra $C\left(X_{A}\right)$ of all complex-valued continuous functions on $X_{A}$ through the correspondence $S_{\mu} S_{\mu}^{*} \in \mathfrak{D}_{A} \longleftrightarrow \chi_{\mu} \in C\left(X_{A}\right)$, where $\chi_{\mu}$ denotes the characteristic function on $X_{A}$ for the cylinder set $U_{\mu}$ for $\mu \in B_{*}\left(X_{A}\right)$. We identify the subalgebra $\mathfrak{D}_{A}$ with $C\left(X_{A}\right)$. It is well known that the algebra $\mathfrak{D}_{A}$ is maximal commutative in $\mathcal{O}_{A}$ ([9, Remark 2.18]; cf. [19, Proposition 3.3]).

For $x=\left(x_{n}\right)_{n \in \mathbb{N}} \in X_{A}$, the orbit $\operatorname{orb}_{\sigma_{A}}(x)$ of $x$ under $\sigma_{A}$ is defined by

$$
\operatorname{orb}_{\sigma_{A}}(x)=\bigcup_{k=0}^{\infty} \bigcup_{l=0}^{\infty} \sigma_{A}^{-k}\left(\sigma_{A}^{l}(x)\right) \subset X_{A} .
$$

Let $\left(X_{A}, \sigma_{A}\right)$ and $\left(X_{B}, \sigma_{B}\right)$ be two topological Markov shifts. If there exists a homeomorphism $h: X_{A} \rightarrow X_{B}$ such that $h\left(\operatorname{orb}_{\sigma_{A}}(x)\right)=\operatorname{orb}_{\sigma_{B}}(h(x))$ for $x \in X_{A}$, then $\left(X_{A}, \sigma_{A}\right)$ and $\left(X_{B}, \sigma_{B}\right)$ are said to be topologically orbit equivalent. In this case, for $x \in X_{A}$, one has $h\left(\sigma_{A}(x)\right) \in \bigcup_{k=0}^{\infty} \bigcup_{l=0}^{\infty} \sigma_{B}^{-k}\left(\sigma_{B}^{l}(h(x))\right)$. Hence there exist $k_{1}, l_{1}: X_{A} \rightarrow \mathbb{Z}_{+}$such that $\sigma_{B}^{k_{1}(x)}\left(h\left(\sigma_{A}(x)\right)\right)=\sigma_{B}^{l_{1}(x)}(h(x))$. Similarly there exist $k_{2}, l_{2}: X_{B} \rightarrow \mathbb{Z}_{+}$such that $\sigma_{A}^{k_{2}(y)}\left(h^{-1}\left(\sigma_{B}(y)\right)\right)=\sigma_{A}^{l_{2}(y)}\left(h^{-1}(y)\right)$. If we may take $k_{1}, l_{1}: X_{A} \longrightarrow \mathbb{Z}_{+}$and $k_{2}, l_{2}: X_{B} \longrightarrow \mathbb{Z}_{+}$as continuous maps, the topological Markov shifts $\left(X_{A}, \sigma_{A}\right)$ and $\left(X_{B}, \sigma_{B}\right)$ are said to be continuously orbit equivalent. In [21, the following has been proved.

Proposition 2.1 ([21, Theorem 5.7]). Let $A$ and $B$ be two irreducible matrices with entries in $\{0,1\}$ satisfying condition (I). There exists an isomorphism $\Psi: \mathcal{O}_{A} \rightarrow$ $\mathcal{O}_{B}$ such that $\Psi\left(\mathfrak{D}_{A}\right)=\mathfrak{D}_{B}$ if and only if $\left(X_{A}, \sigma_{A}\right)$ and $\left(X_{B}, \sigma_{B}\right)$ are continuously orbit equivalent. 


\section{3. $K$-THEORETIC GROUPS}

We assume that an $N \times N$ matrix $A=[A(i, j)]_{i, j=1}^{N}$ with entries in $\{0,1\}$ is irreducible and satisfies condition (I) in the sense of [9]. Let us define the normalizer semigroup of $\mathfrak{D}_{A}$ in $\mathcal{O}_{A}$ by

$$
N_{s}\left(\mathcal{O}_{A}, \mathfrak{D}_{A}\right)=\left\{v \in \mathcal{O}_{A} \mid v \text { is a partial isometry } ; v \mathfrak{D}_{A} v^{*} \subset \mathfrak{D}_{A}, v^{*} \mathfrak{D}_{A} v \subset \mathfrak{D}_{A}\right\} .
$$

It is easy to see that $N_{S}\left(\mathcal{O}_{A}, \mathfrak{D}_{A}\right)$ has a natural structure of an inverse semigroup. Let $\tau: U \rightarrow V$ be a homeomorphism from a clopen set $U \subset X_{A}$ onto a clopen set $V \subset X_{A}$. We call $\tau$ a partial homeomorphism of $X_{A}$. Let us denote by $X_{A, D(\tau)}$ and $X_{A, R(\tau)}$ the clopen sets $U$ and $V$, respectively. We denote by $P H\left(X_{A}\right)$ the set of all partial homeomorphisms of $X_{A}$. Then $P H\left(X_{A}\right)$ has a natural structure of an inverse semigroup (cf. 25], 27]). Let $\left[\sigma_{A}\right]_{s}$ be the set of all partial homeomorphisms $\tau \in P H\left(X_{A}\right)$ such that $\tau(x) \in \operatorname{orb}_{\sigma_{A}}(x)$ for all $x \in X_{A, D(\tau)}$. Let $\left[\sigma_{A}\right]_{s c}$ be the set of all $\tau$ in $\left[\sigma_{A}\right]_{s}$ such that there exist continuous maps $k, l: X_{A, D(\tau)} \rightarrow \mathbb{Z}_{+}$satisfying

$$
\sigma_{A}^{k(x)}(\tau(x))=\sigma_{A}^{l(x)}(x) \quad \text { for all } x \in X_{A, D(\tau)} .
$$

We call $\left[\sigma_{A}\right]_{s c}$ the continuous full inverse semigroup for $\left(X_{A}, \sigma_{A}\right)$. It is clear that $\left[\sigma_{A}\right]_{s}$ is a subsemigroup of $P H\left(X_{A}\right)$ and $\left[\sigma_{A}\right]_{s c}$ is a subsemigroup of $\left[\sigma_{A}\right]_{s}$.

Proposition 3.1 ([20, Proposition 6.4]). For $\tau \in\left[\sigma_{A}\right]_{s c}$, there exists a partial isometry $u_{\tau} \in N_{s}\left(\mathcal{O}_{A}, \mathfrak{D}_{A}\right)$ such that

$$
\begin{array}{ll}
A d\left(u_{\tau}\right)(f)=f \circ \tau^{-1} & \text { for } f \in C\left(X_{A, D(\tau)}\right), \\
A d\left(u_{\tau}^{*}\right)(g)=g \circ \tau & \text { for } g \in C\left(X_{A, R(\tau)}\right)
\end{array}
$$

and the correspondence $\tau \in\left[\sigma_{A}\right]_{s c} \longrightarrow u_{\tau} \in N_{s}\left(\mathcal{O}_{A}, \mathfrak{D}_{A}\right)$ is a homomorphism of inverse semigroups.

We henceforth denote by $\operatorname{Proj}(\mathcal{A})$ the set of projections in a $C^{*}$-algebra $\mathcal{A}$. For $e, f \in \operatorname{Proj}\left(\mathfrak{D}_{A}\right)$, we write $e \widetilde{\mathfrak{D}_{A}} f$ if there exists a partial isometry $v \in$ $N_{s}\left(\mathcal{O}_{A}, \mathfrak{D}_{A}\right)$ such that $e=v^{*} v, v v^{*}=f$. The relation $\sim$ is an equivalence relation in $\operatorname{Proj}\left(\mathfrak{D}_{A}\right)$. We note that if there exists $u \in N_{s}\left(\mathcal{O}_{A}, \mathfrak{D}_{A}\right)$ such that $u^{*} u \geq e$, then $e=(u e)^{*}(u e) \underset{\mathfrak{D}_{A}}{\sim} u e u^{*}$. For two projections $p, q$ in a $C^{*}$-algebra, we write $p \perp q$ if $p q=0$.

\section{Lemma 3.2.}

(i) For $e, f \in \operatorname{Proj}\left(\mathfrak{D}_{A}\right)$, there exists $e^{\prime} \in \operatorname{Proj}\left(\mathfrak{D}_{A}\right)$ such that $e \underset{\mathfrak{D}_{A}}{\sim} e^{\prime} \leq f$.

(ii) For $p_{1}, \ldots, p_{n} \in \operatorname{Proj}\left(\mathfrak{D}_{A}\right)$, there exist $q_{1}, \ldots, q_{n} \in \operatorname{Proj}\left(\mathfrak{D}_{A}\right)$ such that

$$
p_{i} \underset{\mathfrak{D}_{A}}{\sim} q_{i} \text { for } i=1, \ldots, n \text { and } q_{i} \perp q_{j} \text { for } i \neq j .
$$

Proof. (i) One may assume that the projections $e, f$ are written as

$$
e=\sum_{i=1}^{K} \chi_{U_{\mu(i)}}, \quad f=\sum_{j=1}^{L} \chi_{U_{\nu(j)}},
$$

where $\mu(i) \in B_{k}\left(X_{A}\right), i=1, \ldots, K$ for some $k$ and $\nu(j) \in B_{l}\left(X_{A}\right), j=1, \ldots, L$ for some $l$. Since $A$ satisfies condition (I), there exist $J \in\{1, \ldots, N\}$ and words $\xi(1), \ldots, \xi(K) \in B_{*}\left(X_{A}\right)$ with the same length such that the words $\nu(1) \xi(i) J$ are admissible for $i=1, \ldots, K$. Since $A$ is irreducible, there exist words $\eta(1), \ldots, \eta(K)$ 
such that $J \eta(i) \mu(i) \in B_{*}\left(X_{A}\right)$ for $i=1, \ldots, K$. Define a partial homeomorphism $\tau$ of $X_{A}$ by

$$
\tau\left(x_{1}, x_{2}, \ldots\right)=\left(\nu(1), \xi(i), J, \eta(i), x_{1}, x_{2}, \ldots\right) \text { if } x_{1} x_{2} \ldots x_{K}=\mu(i) \text { for some } i .
$$

Then $\tau$ defines an element of $\left[\sigma_{A}\right]_{s c}$ so that a partial isometry $u_{\tau}$ gives rise to an element of $N_{s}\left(\mathcal{O}_{A}, \mathfrak{D}_{A}\right)$. Since $\tau\left(\bigcup_{i=1}^{K} U_{\mu(i)}\right) \subset U_{\nu(1)}$, one has $u_{\tau} e u_{\tau}^{*} \leq f$ so that $e \underset{\mathfrak{D}_{A}}{\sim} u_{\tau} e u_{\tau}^{*} \leq f$

(ii) Let $p_{i}=\sum_{j=1}^{K_{i}} \chi_{U_{\mu_{i}(j)}}$ for $i=1, \ldots, n$ where $\mu_{i}(j) \in B_{*}\left(X_{A}\right)$. Take $k \in \mathbb{N}$ such that $\left|B_{k}\left(X_{A}\right)\right|>n$ and hence there exist $n$ different words $\xi_{1}, \ldots, \xi_{n} \in B_{k}\left(X_{A}\right)$ of length $k$. Since $A$ is irreducible, there exist $\eta_{i}(j) \in B_{*}\left(X_{A}\right), j=1, \ldots, K_{i}$ such that $\xi_{i} \eta_{i}(j) \mu_{i}(j) \in B_{*}\left(X_{A}\right)$ for $i=1, \ldots, n$. For $i=1, \ldots, n$, define a partial homeomorphism $\tau_{i}$ on $X_{A}$ by setting

$$
\tau_{i}\left(x_{1}, x_{2}, \ldots\right)=\left(\xi_{i}, \eta_{i}(j), x_{1}, x_{2}, \ldots\right) \text { for }\left(x_{1}, x_{2}, \ldots\right) \in U_{\mu_{i}(j)} .
$$

Then $\tau_{i} \in\left[\sigma_{A}\right]_{s c}$ such that $\tau_{i}\left(\bigcup_{j=1}^{K_{i}} U_{\mu_{i}(j)}\right)=\bigcup_{j=1}^{K_{i}} U_{\xi_{i} \eta_{i}(j) \mu_{i}(j)}$. Put $q_{i}=$ $\chi_{\cup_{j=1}^{K_{i}} U_{\xi_{i} \eta_{i}(j) \mu_{i}(j)}}$ so that $q_{i} \perp q_{j}$ for $i \neq j$. Take $u_{\tau_{i}} \in N_{s}\left(\mathcal{O}_{A}, \mathfrak{D}_{A}\right)$ such that

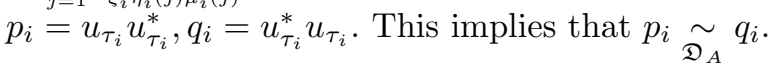

For $p \in \operatorname{Proj}\left(\mathfrak{D}_{A}\right)$, denote by $[p]_{\mathfrak{D}_{A}}$ the equivalence class of $p \in \operatorname{Proj}\left(\mathfrak{D}_{A}\right)$ under the equivalence relation $\widetilde{\mathfrak{D}_{A}}$. For $p, q \in \operatorname{Proj}\left(\mathfrak{D}_{A}\right)$, one may take $p^{\prime}, q^{\prime} \in \operatorname{Proj}\left(\mathfrak{D}_{A}\right)$ by Lemma 3.2 (ii) such that $p \underset{\mathfrak{D}_{A}}{\sim} p^{\prime} \perp q^{\prime} \underset{\mathfrak{D}_{A}}{\sim} q$, so that we define

$$
[p]_{\mathfrak{D}_{A}}+[q]_{\mathfrak{D}_{A}}=\left[p^{\prime}+q^{\prime}\right]_{\mathfrak{D}_{A}} .
$$

We set

$$
K_{0}\left(\mathcal{O}_{A} ; \mathfrak{D}_{A}\right)=\left\{[p]_{\mathfrak{D}_{A}} \mid p \in \operatorname{Proj}\left(\mathfrak{D}_{A}\right)\right\}
$$

Then we have

Lemma 3.3. $K_{0}\left(\mathcal{O}_{A} ; \mathfrak{D}_{A}\right)$ becomes an abelian group under the addition defined by (3.2).

Proof. The proof is similar to the proof of [7, 1.4 Theorem].

Lemma 3.4. For $\mu=\mu_{1} \cdots \mu_{k} \in B_{*}\left(X_{A}\right)$, we have $S_{\mu} S_{\mu}^{*} \widetilde{\mathfrak{D}_{A}} S_{\mu_{k}} S_{\mu_{k}}^{*}$.

Proof. The assertion is obvious by the relations

$$
S_{\mu} S_{\mu}^{*} \underset{\mathfrak{D}_{A}}{\sim} S_{\mu}^{*} S_{\mu}=S_{\mu_{k}}^{*} S_{\mu_{k}} \underset{\mathfrak{D}_{A}}{\sim} S_{\mu_{k}} S_{\mu_{k}}^{*} .
$$

Lemma 3.5. The group $K_{0}\left(\mathcal{O}_{A} ; \mathfrak{D}_{A}\right)$ is generated by $\left[S_{\mu_{1}} S_{\mu_{1}}^{*}\right]_{\mathfrak{D}_{A}}, \ldots,\left[S_{\mu_{k}} S_{\mu_{k}}^{*}\right]_{\mathfrak{D}_{A}}$.

Proof. Since a clopen set of $X_{A}$ is a finite disjoint union of cylinder sets of $X_{A}$, every projection in $\mathfrak{D}_{A}$ is a finite sum of the projections of the form $S_{\mu} S_{\mu}^{*}, \mu \in B_{*}\left(X_{A}\right)$. Hence the assertion holds by the preceding lemma.

We will see that the group $K_{0}\left(\mathcal{O}_{A} ; \mathfrak{D}_{A}\right)$ is canonically isomorphic to the $K_{0}$-group $K_{0}\left(\mathcal{O}_{A}\right)$ of $\mathcal{O}_{A}$. We are now assuming that the matrix $A$ is irreducible satisfying condition (I), so that the algebra $\mathcal{O}_{A}$ is purely infinite and simple. Recall that $K_{0}\left(\mathcal{O}_{A}\right)$ is realized as the abelian group of the equivalence classes $[p]$ of projections $p \in \operatorname{Proj}\left(\mathcal{O}_{A}\right)$, where $p, q \in \operatorname{Proj}\left(\mathcal{O}_{A}\right)$ are equivalent if there exists a partial isometry 
$v \in \mathcal{O}_{A}$ such that $p=v^{*} v, q=v v^{*}$ (see [7]). The addition $[p]+[q]$ for $p, q \in$ $\operatorname{Proj}\left(\mathcal{O}_{A}\right)$ is defined similarly to $(3.2)$. Let $\epsilon_{i}=[0, \ldots, 0, \stackrel{i}{1}, 0, \ldots, 0], i=1, \ldots, N$ be the standard basis for $\mathbb{Z}^{N}$.

Proposition 3.6. The correspondence

$$
\left[S_{i} S_{i}^{*}\right]_{\mathfrak{D}_{A}} \in K_{0}\left(\mathcal{O}_{A} ; \mathfrak{D}_{A}\right) \longrightarrow\left[\epsilon_{i}\right] \in \mathbb{Z}^{N} /\left(1-A^{t}\right) \mathbb{Z}^{N}
$$

gives rise to an isomorphism from the abelian group $K_{0}\left(\mathcal{O}_{A} ; \mathfrak{D}_{A}\right)$ to the quotient group $\mathbb{Z}^{N} /\left(1-A^{t}\right) \mathbb{Z}^{N}$.

Proof. By [6, 3.1 Proposition], the classes $\left[S_{i} S_{i}^{*}\right], i=1, \ldots, N$ generate $K_{0}\left(\mathcal{O}_{A}\right)$ and the correspondence

$$
\delta:\left[S_{i} S_{i}^{*}\right] \in K_{0}\left(\mathcal{O}_{A}\right) \longrightarrow\left[\epsilon_{i}\right] \in \mathbb{Z}^{N} /\left(1-A^{t}\right) \mathbb{Z}^{N}
$$

gives rise to an isomorphism. By the preceding lemma, the correspondences

$$
\begin{aligned}
& \gamma: \epsilon_{i} \in \mathbb{Z}^{N} \longrightarrow\left[S_{i} S_{i}^{*}\right]_{\mathfrak{D}_{A}} \in K_{0}\left(\mathcal{O}_{A} ; \mathfrak{D}_{A}\right), \\
& \eta:\left[S_{i} S_{i}^{*}\right]_{\mathfrak{D}_{A}} \in K_{0}\left(\mathcal{O}_{A} ; \mathfrak{D}_{A}\right) \longrightarrow\left[S_{i} S_{i}^{*}\right] \in K_{0}\left(\mathcal{O}_{A}\right)
\end{aligned}
$$

yield surjective homomorphisms. Denote by

$$
\bar{\gamma}:\left[\epsilon_{i}\right] \in \mathbb{Z}^{N} / \operatorname{Ker}(\gamma) \longrightarrow\left[S_{i} S_{i}^{*}\right]_{\mathfrak{D}_{A}} \in K_{0}\left(\mathcal{O}_{A} ; \mathfrak{D}_{A}\right)
$$

the isomorphism induced by (3.5). As we have

$$
\left[S_{i} S_{i}^{*}\right]_{\mathfrak{D}_{A}}=\left[S_{i}^{*} S_{i}\right]_{\mathfrak{D}_{A}}=\sum_{j=1}^{N} A(i, j)\left[S_{j} S_{j}^{*}\right]_{\mathfrak{D}_{A}},
$$

it follows that $\gamma\left(\epsilon_{i}\right)=\sum_{j=1}^{N} A(i, j) \gamma\left(\epsilon_{j}\right)$ so that $\gamma\left(\epsilon_{i}-\sum_{j=1}^{N} A(i, j) \epsilon_{j}\right)=0$. This implies that $\gamma\left(\epsilon_{i}-A^{t} \epsilon_{i}\right)=0$ for $i=1, \ldots, N$. Hence we have

$$
\gamma\left(\left(1-A^{t}\right) \mathbb{Z}^{N}\right)=0
$$

and $\operatorname{Ker}(\gamma)$ contains $\left(1-A^{t}\right) \mathbb{Z}^{N}$. The natural map

$$
\xi:\left[\epsilon_{i}\right] \in \mathbb{Z}^{N} /\left(1-A^{t}\right) \mathbb{Z}^{N} \longrightarrow\left[\epsilon_{i}\right] \in \mathbb{Z}^{N} / \operatorname{Ker}(\gamma)
$$

gives rise to a surjective homomorphism. We have compositions of surjective homomorphisms

$$
\begin{aligned}
\delta \circ \eta \circ \bar{\gamma} \circ \xi:\left[\epsilon_{i}\right] \in \mathbb{Z}^{N} /\left(1-A^{t}\right) \mathbb{Z}^{N} & \stackrel{\xi}{\longrightarrow}\left[\epsilon_{i}\right] \in \mathbb{Z}^{N} / \operatorname{Ker}(\gamma) \\
& \stackrel{\bar{\gamma}}{\longrightarrow}\left[S_{i} S_{i}^{*}\right]_{\mathfrak{D}_{A}} \in K_{0}\left(\mathcal{O}_{A} ; \mathfrak{D}_{A}\right) \\
& \stackrel{\eta}{\longrightarrow}\left[S_{i} S_{i}^{*}\right] \in K_{0}\left(\mathcal{O}_{A}\right) \\
& \stackrel{\delta}{\longrightarrow}\left[\epsilon_{i}\right] \in \mathbb{Z}^{N} /\left(1-A^{t}\right) \mathbb{Z}^{N} .
\end{aligned}
$$

Since $\bar{\gamma} \circ \xi: \mathbb{Z}^{N} /\left(1-A^{t}\right) \mathbb{Z}^{N} \longrightarrow K_{0}\left(\mathcal{O}_{A} ; \mathfrak{D}_{A}\right)$ is a surjective homomorphism, it gives rise to an isomorphism.

Denote by $\mathcal{K}$ the $C^{*}$-algebra of compact operators on the separable Hilbert space $l^{2}(\mathbb{N})$ of square summable complex sequences on $\mathbb{N}$ and by $\mathcal{C}$ the commutative $C^{*}$ subalgebra of diagonal operators on $l^{2}(\mathbb{N})$. We identify $\mathcal{C}$ with the commutative algebra $c_{0}(\mathbb{N})$ of all complex sequences $\left(c_{n}\right)_{n \in \mathbb{N}}$ convergent to 0 . We set the $C^{*}$ algebras of the tensor products:

$$
\overline{\mathcal{O}}_{A}=\mathcal{O}_{A} \otimes \mathcal{K}, \quad \overline{\mathfrak{D}}_{A}=\mathfrak{D}_{A} \otimes \mathcal{C} .
$$


The set $\operatorname{Proj}\left(\overline{\mathfrak{D}}_{A}\right)$ of projections in $\overline{\mathfrak{D}}_{A}$ is identified with the set $c_{0}\left(\mathbb{N}, \operatorname{Proj}\left(\mathfrak{D}_{A}\right)\right)$ of projection-valued sequences $\left(p_{n}\right)_{n \in \mathbb{N}}$ with finite support. That is,

$$
\begin{aligned}
\operatorname{Proj}\left(\overline{\mathfrak{D}}_{A}\right) & =c_{0}\left(\mathbb{N}, \operatorname{Proj}\left(\mathfrak{D}_{A}\right)\right) \\
& =\left\{\left(p_{n}\right)_{n \in \mathbb{N}} \mid p_{n} \in \operatorname{Proj}\left(\mathfrak{D}_{A}\right), \text { there exists } L \in \mathbb{N} ; p_{n}=0 \text { for } n>L\right\} .
\end{aligned}
$$

We set the normalizer semigroup

$$
N_{s}\left(\overline{\mathcal{O}}_{A}, \overline{\mathfrak{D}}_{A}\right)=\left\{v \in \overline{\mathcal{O}}_{A} \mid v \text { is a partial isometry; } v \overline{\mathfrak{D}}_{A} v^{*} \subset \overline{\mathfrak{D}}_{A}, v^{*} \overline{\mathfrak{D}}_{A} v \subset \overline{\mathfrak{D}}_{A}\right\}
$$

of partial isometries in $\overline{\mathcal{O}}_{A}$. It is easy to see that $N_{s}\left(\overline{\mathcal{O}}_{A}, \overline{\mathfrak{D}}_{A}\right)$ has a natural structure of an inverse semigroup. Put the projection

$$
1_{n}=(\overbrace{1_{A}, \ldots, 1_{A}}^{n}, 0,0, \ldots) \in c_{0}\left(\mathbb{N}, \operatorname{Proj}\left(\mathfrak{D}_{A}\right)\right), \quad n \in \mathbb{N} .
$$

For $v \in N_{s}\left(\overline{\mathcal{O}}_{A}, \overline{\mathfrak{D}}_{A}\right)$ one sees that

$$
v v^{*}=\lim _{n \rightarrow \infty} v 1_{n} v^{*}, \quad v^{*} v=\lim _{n \rightarrow \infty} v^{*} 1_{n} v
$$

so that $v v^{*}, v^{*} v \in \operatorname{Proj}\left(\overline{\mathfrak{D}}_{A}\right)$.

Similarly to the equivalence relation $\widetilde{\mathfrak{D}_{A}} \operatorname{in} \operatorname{Proj}\left(\mathfrak{D}_{A}\right)$, we define an equivalence relation $\underset{\overline{\mathfrak{D}}_{A}}{\sim} \operatorname{in} \operatorname{Proj}\left(\overline{\mathfrak{D}}_{A}\right)$ as follows. For $p, q \in \operatorname{Proj}\left(\overline{\mathfrak{D}}_{A}\right)$, we write $p \underset{\tilde{\mathfrak{D}}_{A}}{\sim} q$ if there exists a partial isometry $v \in N_{s}\left(\overline{\mathcal{O}}_{A}, \overline{\mathfrak{D}}_{A}\right)$ such that $p=v^{*} v, v v^{*}=q$.

Lemma 3.7. For $p=\left(p_{n}\right)_{n \in \mathbb{N}} \in \operatorname{Proj}\left(\overline{\mathfrak{D}}_{A}\right)$ and $K \in \mathbb{N}$, put $p^{\prime}=\left(p_{n+K}\right)_{n \in \mathbb{N}} \in$ $\operatorname{Proj}\left(\overline{\mathfrak{D}}_{A}\right)$. Then we have $p \underset{\overline{\mathfrak{D}}_{A}}{\sim} p^{\prime}$ in $\overline{\mathfrak{D}}_{A}$.

Proof. Let $L \in \mathbb{N}$ be a number satisfying $p_{n}=0$ for all $n>L$. Consider the shift matrix $S$ of size $L+K$,

$$
S=\left[\begin{array}{cccc}
0 & 1 & & \\
\vdots & & \ddots & \\
0 & & & 1 \\
1 & 0 & \ldots & 0
\end{array}\right] \in M_{L+K}(\mathbb{C})
$$

We may regard the matrix algebra $M_{L+K}(\mathbb{C})$ as the subalgebra of $\mathcal{K}$ on the first $L+K$ coordinates on $l^{2}(\mathbb{N})$, so that the element $1 \otimes S$ in $\mathcal{O}_{A} \otimes \mathcal{K}$. Put $p^{\prime}=S^{K} p S^{* K}$ and hence we have $p \underset{\overline{\mathfrak{D}}_{A}}{\sim} p^{\prime}$ in $\overline{\mathfrak{D}}_{A}$.

Lemma 3.8. For $p, q \in \operatorname{Proj}\left(\overline{\mathfrak{D}}_{A}\right)$, there exist $p^{\prime}, q^{\prime} \in \operatorname{Proj}\left(\overline{\mathfrak{D}}_{A}\right)$ such that

$$
p \underset{\overline{\mathfrak{D}}_{A}}{\sim} p^{\prime} \perp q^{\prime} \underset{\widetilde{\mathfrak{D}}_{A}}{\sim} q
$$

Proof. Assume that $p=\left(p_{n}\right)_{n \in \mathbb{N}}, q=\left(q_{n}\right)_{n \in \mathbb{N}} \in \operatorname{Proj}\left(\overline{\mathfrak{D}}_{A}\right)$. Take $L \in \mathbb{N}$ such that $q_{n}=0$ for all $n>L$. By the preceding lemma, the projection $p^{\prime}=\left(p_{n+L}\right)_{n \in \mathbb{N}}$ is equivalent to $p$ and perpendicular to $q$.

Lemma 3.9. For a projection $p \in \operatorname{Proj}\left(\overline{\mathfrak{D}}_{A}\right)$, there exists a projection $e \in \operatorname{Proj}\left(\mathfrak{D}_{A}\right)$ such that

$$
p \underset{\overline{\mathfrak{D}}_{A}}{\sim} p_{e}, \quad \text { where } \quad p_{e}=(e, 0,0, \ldots) \in \operatorname{Proj}\left(\overline{\mathfrak{D}}_{A}\right)
$$


Proof. For $p=\left(p_{1}, \ldots, p_{n}, 0, \ldots\right) \in c_{0}\left(\mathbb{N}, \operatorname{Proj}\left(\mathfrak{D}_{A}\right)\right)$, by Lemma 3.2 (ii) there exist $q_{1}, \ldots, q_{n} \in \operatorname{Proj}\left(\mathfrak{D}_{A}\right)$ such that

$$
p_{i} \underset{\mathfrak{D}_{A}}{\sim} q_{i} \text { for } i=1, \ldots, n \text { and } q_{i} \perp q_{j} \text { for } i \neq j .
$$

Put $e=q_{1}+\cdots+q_{n} \in \operatorname{Proj}\left(\mathfrak{D}_{A}\right)$. As $p_{i} \underset{\mathfrak{D}_{A}}{\sim} q_{i}$ for $i=1, \ldots, n$, it is easy to see that $p \underset{\tilde{\mathfrak{D}}_{A}}{\sim} p_{e}$.

For $p \in \operatorname{Proj}\left(\overline{\mathfrak{D}}_{A}\right)$, denote by $[p]_{\overline{\mathfrak{D}}_{A}}$ the equivalence class of $p \in \operatorname{Proj}\left(\overline{\mathfrak{D}}_{A}\right)$ under the equivalence relation $\underset{\overline{\mathfrak{D}}_{A}}{\sim}$. For $p, q \in \operatorname{Proj}\left(\overline{\mathfrak{D}}_{A}\right)$, take $p^{\prime}, q^{\prime} \in \operatorname{Proj}\left(\overline{\mathfrak{D}}_{A}\right)$ such that $p \underset{\mathfrak{D}_{A}}{\sim} p^{\prime} \perp q^{\prime} \underset{\overline{\mathfrak{D}}_{A}}{\sim} q$. We then define

$$
[p]_{\overline{\mathfrak{D}}_{A}}+[q]_{\overline{\mathfrak{D}}_{A}}=\left[p^{\prime}+q^{\prime}\right]_{\overline{\mathfrak{D}}_{A}} .
$$

We set

$$
K_{0}\left(\overline{\mathcal{O}}_{A} ; \overline{\mathfrak{D}}_{A}\right)=\left\{[p]_{\overline{\mathfrak{D}}_{A}} \mid p \in \operatorname{Proj}\left(\overline{\mathfrak{D}}_{A}\right)\right\}
$$

Then we have

Lemma 3.10. $K_{0}\left(\overline{\mathcal{O}}_{A} ; \overline{\mathfrak{D}}_{A}\right)$ becomes an abelian group under the addition defined by (3.7).

Proof. It is clear that the definition (3.7) is independent of the choice of $p^{\prime}, q^{\prime} \in$ $\operatorname{Proj}\left(\overline{\mathfrak{D}}_{A}\right)$ satisfying $p \underset{\overline{\mathfrak{D}}_{A}}{\sim} p^{\prime} \perp q^{\prime} \underset{\overline{\mathfrak{D}}_{A}}{\sim} q$.

\section{Lemma 3.11.}

(i) The group $K_{0}\left(\overline{\mathcal{O}}_{A} ; \overline{\mathfrak{D}}_{A}\right)$ is generated by the classes of the projections $p_{e}=$ $(e, 0, \ldots) \in \operatorname{Proj}\left(\overline{\mathfrak{D}}_{A}\right)$ for $e \in \operatorname{Proj}\left(\mathfrak{D}_{A}\right)$.

(ii) The correspondence

$$
\left[p_{e}\right]_{\overline{\mathfrak{D}}_{A}} \in K_{0}\left(\overline{\mathcal{O}}_{A} ; \overline{\mathfrak{D}}_{A}\right) \longrightarrow[e]_{\mathfrak{D}_{A}} \in K_{0}\left(\mathcal{O}_{A} ; \mathfrak{D}_{A}\right)
$$

gives rise to an isomorphism from $K_{0}\left(\overline{\mathcal{O}}_{A} ; \overline{\mathfrak{D}}_{A}\right)$ to $K_{0}\left(\mathcal{O}_{A} ; \mathfrak{D}_{A}\right)$.

Proof. (i) The assertion comes from Lemma 3.9.

(ii) It suffices to show that $\left[p_{e}\right]_{\overline{\mathfrak{D}}_{A}}=\left[p_{f}\right]_{\overline{\mathfrak{D}}_{A}}$ implies $[e]_{\mathfrak{D}_{A}}=[f]_{\mathfrak{D}_{A}}$. Suppose that $\left[p_{e}\right]_{\overline{\mathfrak{D}}_{A}}=\left[p_{f}\right]_{\mathfrak{\mathfrak { D }}_{A}}$ for some $e, f \in \operatorname{Proj}\left(\mathfrak{D}_{A}\right)$. There exists a partial isometry $v \in N_{s}\left(\overline{\mathcal{O}}_{A}, \overline{\mathfrak{D}}_{A}\right)$ such that $v^{*} v=p_{e}, v v^{*}=p_{f}$. Denote by $1_{1}=\left(1_{A}, 0, \ldots\right) \in$ $\operatorname{Proj}\left(\overline{\mathfrak{D}}_{A}\right) \subset \operatorname{Proj}\left(\overline{\mathcal{O}}_{A}\right)$. Since $1_{1} v^{*} v=v^{*} v=v^{*} v 1_{1}$ and $1_{1} v v^{*}=v v^{*}=v v^{*} 1_{1}$, by putting $u=1_{1} v 1_{1}$, we have $u^{*} u=p_{e}, u u^{*}=p_{f}$. As $u$ is regarded as an element of $N_{s}\left(\mathcal{O}_{A}, \mathfrak{D}_{A}\right)$, we have $[e]_{\mathfrak{D}_{A}}=[f]_{\mathfrak{D}_{A}}$ in $K_{0}\left(\mathcal{O}_{A} ; \mathfrak{D}_{A}\right)$.

Therefore we have

\section{Proposition 3.12.}

(i) The correspondence

$$
[p]_{\overline{\mathfrak{D}}_{A}} \in K_{0}\left(\overline{\mathcal{O}}_{A} ; \overline{\mathfrak{D}}_{A}\right) \longrightarrow \sum_{i=1}^{n}\left[p_{i}\right]_{\mathfrak{D}_{A}} \in K_{0}\left(\mathcal{O}_{A} ; \mathfrak{D}_{A}\right)
$$

for $p=\left(p_{1}, \ldots, p_{n}, 0, \ldots\right) \in \operatorname{Proj}\left(\overline{\mathfrak{D}}_{A}\right)$ gives rise to an isomorphism. 
(ii) The correspondence

$$
[p]_{\overline{\mathfrak{D}}_{A}} \in K_{0}\left(\overline{\mathcal{O}}_{A} ; \overline{\mathfrak{D}}_{A}\right) \longrightarrow[p] \in K_{0}\left(\overline{\mathcal{O}}_{A}\right)=K_{0}\left(\mathcal{O}_{A}\right)
$$

for $p=\left(p_{1}, \ldots, p_{n}, 0, \ldots\right) \in \operatorname{Proj}\left(\overline{\mathfrak{D}}_{A}\right)$ gives rise to an isomorphism, where $p$ is regarded as an element of $\overline{\mathcal{O}}_{A}$.

Proof. (i) As we have

$$
\begin{aligned}
{[p]_{\overline{\mathfrak{D}}_{A}} } & =\left[\left(p_{1}, p_{2}, \ldots, p_{n}, 0, \ldots\right)\right]_{\overline{\mathfrak{D}}_{A}} \\
& =\left[\left(p_{1}, 0, \ldots\right)\right]_{\overline{\mathfrak{D}}_{A}}+\left[\left(0, p_{2}, 0, \ldots\right)\right]_{\overline{\mathfrak{D}}_{A}}+\cdots+\left[\left(0, \ldots, 0, p_{n}, 0, \ldots\right)\right]_{\overline{\mathfrak{D}}_{A}}
\end{aligned}
$$

by Lemma 3.7 and Lemma 3.11 , the assertion holds.

(ii) The assertion is now obvious.

Therefore we have

Proposition 3.13. Assume that $A$ is an irreducible square matrix with entries in $\{0,1\}$ satisfying condition (I). The correspondence

$$
[p]_{\overline{\mathfrak{D}}_{A}} \in K_{0}\left(\overline{\mathcal{O}}_{A} ; \overline{\mathfrak{D}}_{A}\right) \longrightarrow[p] \in K_{0}\left(\overline{\mathcal{O}}_{A}\right)
$$

for $p \in \operatorname{Proj}\left(\overline{\mathfrak{D}}_{A}\right) \subset \operatorname{Proj}\left(\overline{\mathcal{O}}_{A}\right)$ gives rise to an isomorphism so that we have isomorphisms

$$
K_{0}\left(\overline{\mathcal{O}}_{A} ; \overline{\mathfrak{D}}_{A}\right) \cong K_{0}\left(\mathcal{O}_{A} ; \mathfrak{D}_{A}\right) \cong K_{0}\left(\overline{\mathcal{O}}_{A}\right) \cong \mathbb{Z}^{N} /\left(1-A^{t}\right) \mathbb{Z}^{N}
$$

Corollary 3.14. For $p, q \in \operatorname{Proj}\left(\overline{\mathfrak{D}}_{A}\right) \subset \operatorname{Proj}\left(\overline{\mathcal{O}}_{A}\right)$, we have

$$
[p]_{\overline{\mathfrak{D}}_{A}}=[q]_{\mathfrak{\mathfrak { D }}_{A}} \text { in } K_{0}\left(\overline{\mathcal{O}}_{A} ; \overline{\mathfrak{D}}_{A}\right) \text { if and only if }[p]=[q] \text { in } K_{0}\left(\overline{\mathcal{O}}_{A}\right) \text {. }
$$

\section{MAin RESUlts}

Let us denote by $\left(\bar{X}_{A}, \bar{\sigma}_{A}\right)$ the two-sided topological Markov shift for $A$, where $\bar{X}_{A}$ is the shift space defined by

$$
\bar{X}_{A}=\left\{\left(x_{n}\right)_{n \in \mathbb{Z}} \in\{1, \ldots, N\}^{\mathbb{Z}} \mid A\left(x_{n}, x_{n+1}\right)=1 \text { for all } n \in \mathbb{Z}\right\}
$$

and $\bar{\sigma}_{A}: \bar{X}_{A} \longrightarrow \bar{X}_{A}$ is the homeomorphism of the shift defined by $\bar{\sigma}_{A}\left(\left(x_{n}\right)_{n \in \mathbb{Z}}\right)=$ $\left(x_{n+1}\right)_{n \in \mathbb{Z}}$. Thanks to the discussions on $K$-theoretic groups in the preceding section, we reach the following theorem.

Theorem 4.1. Let $A$ and $B$ be two irreducible square matrices with entries in $\{0,1\}$ satisfying condition (I). Suppose that the equality $\operatorname{det}(1-A)=\operatorname{det}(1-B)$ holds. If there exists an isomorphism $\alpha: K_{0}\left(\mathcal{O}_{A}\right) \longrightarrow K_{0}\left(\mathcal{O}_{B}\right)$ such that $\alpha\left(\left[1_{A}\right]\right)=\left[1_{B}\right]$, then there exists an isomorphism $\Psi: \mathcal{O}_{A} \rightarrow \mathcal{O}_{B}$ such that $\Psi\left(\mathfrak{D}_{A}\right)=\mathfrak{D}_{B}$ and $\Psi_{*}=\alpha$.

Proof. Since $K_{0}\left(\mathcal{O}_{A}\right) \cong K_{0}\left(\mathcal{O}_{B}\right)$ and $\operatorname{det}(1-A)=\operatorname{det}(1-B)$, the two-sided topological Markov shifts $\left(\bar{X}_{A}, \bar{\sigma}_{A}\right)$ and $\left(\bar{X}_{B}, \bar{\sigma}_{B}\right)$ are flow equivalent $([11])$. Hence by [9], 4.1 Theorem], there exists an isomorphism $\varphi: \overline{\mathcal{O}}_{A} \longrightarrow \overline{\mathcal{O}}_{B}$ such that $\varphi\left(\overline{\mathfrak{D}}_{A}\right)=\overline{\mathfrak{D}}_{B}$. Let $e_{1}$ be the rank one projection in $\mathcal{K}$ defined by $e_{1}=(1,0,0, \ldots) \in \mathcal{C}$. Define the isomorphism $\beta=\alpha \circ \varphi_{*}^{-1}: K_{0}\left(\mathcal{O}_{B}\right) \longrightarrow K_{0}\left(\mathcal{O}_{B}\right)$ which satisfies

$$
\beta\left(\left[\varphi\left(1_{A} \otimes e_{1}\right)\right]\right)=\alpha\left(\left[1_{A} \otimes e_{1}\right]\right)=\left[1_{B} \otimes e_{1}\right] .
$$

By Huang's theorem [15, Theorem 2.15] and its proof, any automorphism on $K_{0}\left(\mathcal{O}_{B}\right)$ is induced by a flow equivalence, and the flow equivalence gives rise to an automorphism $\psi$ on $\overline{\mathcal{O}}_{B}$ such that $\psi\left(\overline{\mathfrak{D}}_{B}\right)=\overline{\mathfrak{D}}_{B}$ and $\psi_{*}=\beta$ (see also [6]). We 
then have an isomorphism $\psi \circ \varphi: \overline{\mathcal{O}}_{A} \longrightarrow \overline{\mathcal{O}}_{B}$ such that $\psi \circ \varphi\left(\overline{\mathfrak{D}}_{A}\right)=\overline{\mathfrak{D}}_{B}$. It satisfies

$$
\left[\psi \circ \varphi\left(1_{A} \otimes e_{1}\right)\right]=\beta\left(\left[\varphi\left(1_{A} \otimes e_{1}\right)\right]\right)=\left[1_{B} \otimes e_{1}\right] \quad \text { in } \quad K_{0}\left(\overline{\mathcal{O}}_{B}\right) .
$$

As $\psi \circ \varphi\left(1_{A} \otimes e_{1}\right), 1_{B} \otimes e_{1} \in \operatorname{Proj}\left(\overline{\mathfrak{D}}_{B}\right)$, Corollary 3.14 implies that

$$
\left[\psi \circ \varphi\left(1_{A} \otimes e_{1}\right)\right]_{\overline{\mathfrak{D}}_{B}}=\left[1_{B} \otimes e_{1}\right]_{\overline{\mathfrak{D}}_{B}} \quad \text { in } \quad K_{0}\left(\overline{\mathcal{O}}_{B} ; \overline{\mathfrak{D}}_{B}\right) .
$$

There exists a partial isometry $v \in N_{s}\left(\overline{\mathcal{O}}_{B}, \overline{\mathfrak{D}}_{B}\right)$ such that $v v^{*}=1_{B} \otimes e_{1}, v^{*} v=$ $\psi \circ \varphi\left(1_{A} \otimes e_{1}\right)$. The compositions of the maps

$$
a \otimes e_{1} \in \mathcal{O}_{A} \otimes \mathbb{C} e_{1} \stackrel{\psi \circ \varphi}{\longrightarrow} \psi \circ \varphi\left(a \otimes e_{1}\right) \in \overline{\mathcal{O}}_{B} \stackrel{A d v}{\longrightarrow} v \psi\left(\varphi\left(a \otimes e_{1}\right)\right) v^{*} \in \mathcal{O}_{B} \otimes \mathbb{C} e_{1}
$$

give rise to an isomorphism $A d v \circ \psi \circ \varphi$ from $\mathcal{O}_{A} \otimes \mathbb{C} e_{1}$ to $\mathcal{O}_{B} \otimes \mathbb{C} e_{1}$ such that $A d v \circ \psi \circ \varphi\left(\mathfrak{D}_{A} \otimes \mathbb{C} e_{1}\right)=\mathfrak{D}_{B} \otimes \mathbb{C} e_{1}$. By putting $\Psi=\left.A d v \circ \psi \circ \varphi\right|_{\mathcal{O}_{A} \otimes \mathbb{C} e_{1}}$, one has a desired isomorphism from $\mathcal{O}_{A}$ to $\mathcal{O}_{B}$ which satisfies $\Psi_{*}=\alpha$.

The following lemma is well-known and easily shown.

Lemma 4.2. If $K_{0}\left(\mathcal{O}_{A}\right)$ is isomorphic to $K_{0}\left(\mathcal{O}_{B}\right)$ and $\operatorname{det}(1-A) \times \operatorname{det}(1-B) \geq 0$, then $\operatorname{det}(1-A)=\operatorname{det}(1-B)$.

Proof. Since the groups $K_{0}\left(\mathcal{O}_{A}\right) \cong K_{0}\left(\mathcal{O}_{B}\right)$ are finitely generated abelian groups, they are written as $\mathbb{Z}_{m_{1}} \oplus \cdots \oplus \mathbb{Z}_{m_{r}} \oplus \mathbb{Z}^{k}$ for some nonnegative integers $m_{i}, k \in \mathbb{Z}_{+}$. Then $k \neq 0$ if and only if $\operatorname{det}(1-A)=0$, and hence equivalently $\operatorname{det}(1-B)=0$. If $k=0$, one sees that $|\operatorname{det}(1-A)|=|\operatorname{det}(1-B)|=m_{1} \cdots m_{r}$. Under the condition $\operatorname{det}(1-A) \times \operatorname{det}(1-B) \geq 0$, one has $\operatorname{det}(1-A)=\operatorname{det}(1-B)$.

We note that if there exists an isomorphism from $\mathcal{O}_{A}$ to $\mathcal{O}_{B}$, it induces an isomorphism from $K_{0}\left(\mathcal{O}_{A}\right)$ to $K_{0}\left(\mathcal{O}_{B}\right)$ which maps $\left[1_{A}\right]$ to $\left[1_{B}\right]$. We therefore reach the main theorem.

Theorem 4.3. Let $A$ and $B$ be two irreducible square matrices with entries in $\{0,1\}$ satisfying condition (I). Suppose that the inequality $\operatorname{det}(1-A) \times \operatorname{det}(1-B) \geq 0$ holds. Then the following conditions are equivalent:

(i) The Cuntz-Krieger algebras $\mathcal{O}_{A}$ and $\mathcal{O}_{B}$ are isomorphic.

(ii) The one-sided topological Markov shifts $\left(X_{A}, \sigma_{A}\right)$ and $\left(X_{B}, \sigma_{B}\right)$ are continuously orbit equivalent.

Proof. The implication (i) $\Rightarrow$ (ii) is deduced from Proposition 2.1, Theorem 4.1 and Lemma 4.2. The other implication comes from Proposition 2.1.

Corollary 4.4. Let $A$ and $B$ be two irreducible square matrices with entries in $\{0,1\}$ satisfying condition (I). Suppose that $K_{1}\left(\mathcal{O}_{A}\right) \neq\{0\}$ or $K_{1}\left(\mathcal{O}_{B}\right) \neq\{0\}$. Then the following conditions are equivalent:

(i) The Cuntz-Krieger algebras $\mathcal{O}_{A}$ and $\mathcal{O}_{B}$ are isomorphic.

(ii) The one-sided topological Markov shifts $\left(X_{A}, \sigma_{A}\right)$ and $\left(X_{B}, \sigma_{B}\right)$ are continuously orbit equivalent. 
Proof. It suffices to show the implication (i) $\Rightarrow$ (ii). Suppose that $K_{1}\left(\mathcal{O}_{A}\right) \neq\{0\}$. As $K_{1}\left(\mathcal{O}_{A}\right) \cong \operatorname{Ker}\left(1-A^{t}\right)$ in $\mathbb{Z}^{N}$, we have $\operatorname{det}(1-A)=\operatorname{det}\left(1-A^{t}\right)=0$. Hence the condition $\operatorname{det}(1-A) \times \operatorname{det}(1-B) \geq 0$ in the above theorem is satisfied. Therefore we have the assertion.

Enomoto-Fujii-Watatani in [10 have introduced a notion of primitive equivalence in square matrices with entries in $\{0,1\}$ and in its associated finite directed graphs and proved that the equivalence relation completely classifies the isomorphism classes of the Cuntz-Krieger algebras defined by $3 \times 3$ matrices with entries in $\{0,1\}$. The author in 22 has shown that the equivalence relation preserves the continuous orbit equivalence relation of the associated one-sided toological Markov shifts, so that the isomorphism classes of the Cuntz-Krieger algebras defined by $3 \times 3$ matrices with entries in $\{0,1\}$ are completely classified by the continuous orbit equivalence classes of the associated one-sided topological Markov shifts. As a result, for the matrices whose sizes are less than or equal to three, it has been proved in 22] that the two conditions (i) and (ii) in Theorem 4.3 are equivalent without the hypothesis on the determinants of the matrices. Since the primitive equivalence preserves the determinants [10, Theorem 8.4], one may directly see the following result stated in [22] by using Theorem 4.3 .

Theorem 4.5 (22]). Let $A$ and $B$ be two irreducible square matrices with entries in $\{0,1\}$ satisfying condition (I). Suppose that the sizes of the matrices $A$ and $B$ are less than or equal to three. Then the following conditions are equivalent:

(i) The Cuntz-Krieger algebras $\mathcal{O}_{A}$ and $\mathcal{O}_{B}$ are isomorphic.

(ii) The one-sided topological Markov shifts $\left(X_{A}, \sigma_{A}\right)$ and $\left(X_{B}, \sigma_{B}\right)$ are continuously orbit equivalent.

Proof. It suffices to show the implication (i) $\Longrightarrow$ (ii) for the matrices $A, B$ whose sizes are both less than or equal to three. Suppose that $\mathcal{O}_{A}$ is isomorphic to $\mathcal{O}_{B}$. If $A$ and $B$ are both $3 \times 3$ matrices, they are primitive equivalent to each other by [10, Theorem 4.1]. The equality $\operatorname{det}(1-A)=\operatorname{det}(1-B)$ holds under the primitive equivalence by [10, Theorem 8.4]. Hence condition (ii) comes from Theorem 4.3. If $A$ and $B$ are both $2 \times 2$ matrices, then they are $\left[\begin{array}{ll}1 & 1 \\ 1 & 1\end{array}\right]$ or $\left[\begin{array}{ll}1 & 1 \\ 1 & 0\end{array}\right]$, because of

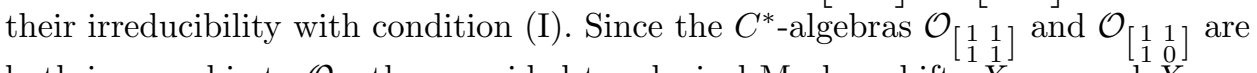

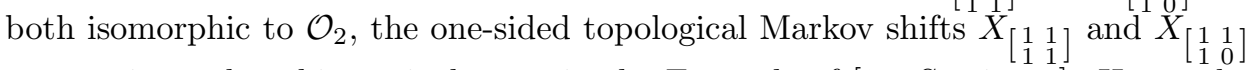
are continuously orbit equivalent as in the Example of [21, Section 5]. Hence the implication (i) $\Longrightarrow$ (ii) holds in this case. We may finally assume that $A$ is a

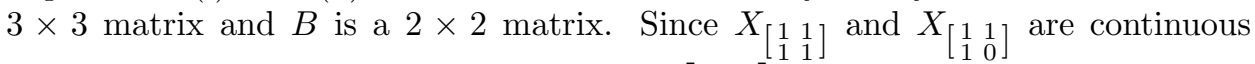
orbit equivalent, we may assume that $B=\left[\begin{array}{ll}1 & 1 \\ 1 & 1\end{array}\right]$. By the hypothesis that $\mathcal{O}_{A}$ is isomorphic to $\mathcal{O}_{B}$, the $3 \times 3$ matrix $A$ is one of the 13 matrices in the classification table [10, p. 450], whose representative is $\mathcal{O}_{2}$. One may take the matrix $A$ as $\left[\begin{array}{lll}0 & 1 & 0 \\ 1 & 1 & 1 \\ 1 & 0 & 1\end{array}\right]$, which is one of the 13 matrices. As the first column and the third column are the same in the matrix $A$, we may consider its column amalgamation matrix, which is $B$ (see [17, §2.1]). This implies that the one-sided topological Markov shifts $\left(X_{A}, \sigma_{A}\right)$ and $\left(X_{B}, \sigma_{B}\right)$ are topologically conjugate and hence continuously orbit equivalent. Therefore the implication (i) $\Longrightarrow$ (ii) holds. 
We remark that there are $4 \times 4$ irreducible matrices $A, B$ with condition (I) such that $\mathcal{O}_{A}$ is isomorphic to $\mathcal{O}_{B}$, whereas $\operatorname{det}(1-A) \neq \operatorname{det}(1-B)([28])$.

\section{Automorphisms on $\mathcal{O}_{A}$ AND orbit Equivalence}

D. Huang's theorem [15, Theorem 2.15] shows that any automorphism on the group $K_{0}\left(\mathcal{O}_{A}\right)$ comes from a flow equivalence on the two-sided topological Markov shift $\left(\bar{X}_{A}, \bar{\sigma}_{A}\right)$. We apply the preceding discussions to study automorphisms on $\mathcal{O}_{A}$ from the viewpoint of orbit equivalence. A homeomorphism $h$ on $X_{A}$ is called an orbit equivalent homeomorphism if there exist continuous maps $k_{1}, l_{1}, k_{2}, l_{2}$ : $X_{A} \rightarrow \mathbb{Z}_{+}$such that

$$
\begin{aligned}
\sigma_{A}^{k_{1}(x)}\left(h\left(\sigma_{A}(x)\right)\right) & =\sigma_{A}^{l_{1}(x)}(h(x)), \\
\sigma_{A}^{k_{2}(x)}\left(h^{-1}\left(\sigma_{A}(x)\right)\right) & =\sigma_{A}^{l_{2}(x)}\left(h^{-1}(x)\right),
\end{aligned}
$$

for all $x \in X_{A}$. We denote by $H_{\sigma_{A}}\left(X_{A}\right)$ the set of all orbit equivalent homeomorphisms on $X_{A}$. The set $H_{\sigma_{A}}\left(X_{A}\right)$ has been written as $N_{c}\left[\sigma_{A}\right]$ in [21]. By 21. Lemma 6.2], it is the normalizer subgroup of the continuous full group $\left[\sigma_{A}\right]_{c}$ in the group of all homeomorphisms on $X_{A}$. Since an orbit equivalent homeomorphism $h \in H_{\sigma_{A}}\left(X_{A}\right)$ gives rise to a continuous orbit equivalence on $\left(X_{A}, \sigma_{A}\right)$, it induces an automorphism $\alpha_{h}$ on $\mathcal{O}_{A}$ satisfying $\alpha_{h}\left(\mathfrak{D}_{A}\right)=\mathfrak{D}_{A}$ ([21, Proposition 6.3]). Let us denote by $\operatorname{Aut}\left(\mathcal{O}_{A}, \mathfrak{D}_{A}\right)$ the group of automorphisms $\alpha$ on $\mathcal{O}_{A}$ satisfying $\alpha\left(\mathfrak{D}_{A}\right)=\mathfrak{D}_{A}$. Then we have

Proposition 5.1. Assume that $A$ is an irreducible square matrix with entries in $\{0,1\}$ satisfying condition (I). For an automorphism $\beta$ on the group $K_{0}\left(\mathcal{O}_{A}\right)$ satisfying $\beta\left(\left[1_{A}\right]\right)=\left[1_{A}\right]$, there exists an orbit equivalent homeomorphism $h \in H_{\sigma_{A}}\left(X_{A}\right)$ such that the induced automorphism $\alpha_{h}$ on $\operatorname{Aut}\left(\mathcal{O}_{A}, \mathfrak{D}_{A}\right)$ satisfies $\alpha_{h *}=\beta$ on $K_{0}\left(\mathcal{O}_{A}\right)$.

Proof. We apply Theorem 4.3 for the matrix $A=B$. There exists an automorphism $\Psi$ on $\mathcal{O}_{A}$ satisfying $\Psi\left(\mathfrak{D}_{A}\right)=\mathfrak{D}_{A}$ and $\Psi_{*}=\beta$ on $K_{0}\left(\mathcal{O}_{A}\right)$. By 21, $\Psi \in \operatorname{Aut}\left(\mathcal{O}_{A}, \mathfrak{D}_{A}\right)$ induces an orbit equivalent homeomorphism $h: X_{A} \longrightarrow X_{A}$. Let $\alpha_{h} \in \operatorname{Aut}\left(\mathcal{O}_{A}, \mathfrak{D}_{A}\right)$ be the induced automorphism from $h \in H_{\sigma_{A}}\left(X_{A}\right)$. Since $\left.\Psi\right|_{\mathfrak{D}_{A}}=\left.\alpha_{h}\right|_{\mathfrak{D}_{A}}=h$ on $X_{A}$, one has

$$
\Psi_{*}\left(\left[S_{i} S_{i}^{*}\right]\right)=\alpha_{h *}\left(\left[S_{i} S_{i}^{*}\right]\right)=\left[h\left(S_{i} S_{i}^{*}\right)\right]
$$

in $K_{0}\left(\mathcal{O}_{A}\right)$. Hence $\Psi_{*}=\alpha_{h *}$ on $K_{0}\left(\mathcal{O}_{A}\right)$. Therefore we have $\alpha_{h *}=\beta$ so that we have the assertion.

It is well-known that any automorphism on $K_{0}\left(\mathcal{O}_{A}\right)$ keeping the class $\left[1_{A}\right]$ of the unit of $\mathcal{O}_{A}$ comes from an automorphism on the algebra $\mathcal{O}_{A}$ (see [28]).

Corollary 5.2. Assume that $A$ is an irreducible square matrix with entries in $\{0,1\}$ satisfying condition (I). For any automorphism $\alpha$ on the Cuntz-Krieger algebra $\mathcal{O}_{A}$, there exists an automorphism $\alpha_{h}$ on $\mathcal{O}_{A}$ induced from a homeomorphism $h$ which gives rise to a continuous orbit equivalence of the one-sided topological Markov shift $\left(X_{A}, \sigma_{A}\right)$ such that $\alpha_{h}\left(\mathfrak{D}_{A}\right)=\mathfrak{D}_{A}$ and $\alpha_{h *}=\alpha_{*}$ on $K_{0}\left(\mathcal{O}_{A}\right)$. 


\section{Concluding REMARKS}

We have used the hypothesis that the determinants of $1-A$ and $1-B$ have the same sign to prove Theorem 1.1 and Theorem 1.2. The hypothesis is needed in our discussions. However we do not know examples of topological Markov shifts $\left(X_{A}, \sigma_{A}\right)$ and $\left(X_{B}, \sigma_{B}\right)$ which are continuously orbit equivalent and satisfy $\operatorname{det}(1-A) \neq \operatorname{det}(1-B)$. We present the following conjecture:

Conjecture: The determinant $\operatorname{det}(1-A)$ is invariant under the continuous orbit equivalence class of $\left(X_{A}, \sigma_{A}\right)$.

If the conjecture is true, the triplet $\left(K_{0}\left(\mathcal{O}_{A}\right),\left[1_{A}\right], \operatorname{det}(1-A)\right)$ would be a complete invariant for the continuous orbit equivalence class of $\left(X_{A}, \sigma_{A}\right)$. Then we would have: the one-sided topological Markov shifts $\left(X_{A}, \sigma_{A}\right)$ and $\left(X_{B}, \sigma_{B}\right)$ are continuously orbit equivalent if and only if the Cuntz-Krieger algebras $\mathcal{O}_{A}$ and $\mathcal{O}_{B}$ are isomorphic and $\operatorname{det}(1-A)=\operatorname{det}(1-B)$.

\section{ACKNOWLEDGMENTS}

The author would like to thank the referee for a careful reading of the first draft of the paper and useful suggestions on an organizational change of lemmas. This work was supported by JSPS Grant-in-Aid for Scientific Research (C), No. 23540237.

\section{REFERENCES}

[1] R. Bowen and J. Franks, Homology for zero-dimensional nonwandering sets, Ann. Math. 106(1977), pp. 73-92. MR0458492 (56:16692)

[2] M. Boyle, Topological orbit equivalence and factor maps in symbolic dynamics, Ph.D. Thesis, University of Washington, 1983. MR2632783

[3] M. Boyle and D. Handelman, Orbit equivalence, flow equivalence and ordered cohomology, Israel J. Math. 95(1996), pp. 169-210. MR1418293 (98a:46082)

[4] M. Boyle and J. Tomiyama, Bounded topological orbit equivalence and $C^{*}$-algebras, J. Math. Soc. Japan 50(1998), pp. 317-329. MR.1613140 (99d:46088)

[5] J. Cuntz, Simple $C^{*}$-algebras generated by isometries, Comm. Math. Phys. 57(1977), pp. 173185. MR0467330 (57:7189)

[6] J. Cuntz, A class of $C^{*}$-algebras and topological Markov chains II: Reducible chains and the Ext-functor for $C^{*}$-algebras, Invent. Math. 63(1981), pp. 25-40. MR608527 (82f:46073b)

[7] J. Cuntz, K-theory for certain $C^{*}$-algebras, Ann. Math. 113(1981), pp. 181-197. MR604046 (84c:46058)

[8] J. Cuntz, The classification problem for the $C^{*}$-algebra $\mathcal{O}_{A}$, Geometric methods in operator algebras, Pitman Research Notes in Mathematics Series 123, Longman Sci. Tech., Harlow (1986), pp. 145-151. MR866492 (88a:46081)

[9] J. Cuntz and W. Krieger, A class of $C^{*}$-algebras and topological Markov chains, Invent. Math. 56(1980), pp. 251-268. MR561974 (82f:46073a)

[10] M. Enomoto, M. Fujii and Y. Watatani, $K_{0}$-groups and classifications of Cuntz-Krieger algebras, Math. Japon. 26(1981), pp. 443-460. MR634920 (83d:46070)

[11] J. Franks, Flow equivalence of subshifts of finite type, Ergodic Theory Dynam. Systems 4(1984), pp. 53-66. MR758893 (86j:58078)

[12] T. Giordano, I. F. Putnam and C. F. Skau, Topological orbit equivalence and $C^{*}$-crossed products, J. reine angew. Math. 469(1995), pp. 51-111. MR.1363826 (97g:46085)

[13] T. Giordano, I. F. Putnam and C. F. Skau, Full groups of Cantor minimal systems, Isr. J. Math. 111(1999), pp. 285-320. MR1710743 (2000g:46096)

[14] T. Giordano, H. Matui, I. F. Putnam and C. F. Skau, Orbit equivalemce for Cantor minimal $\mathbb{Z}^{2}$-systems, J. Amer. Math. Soc. 21(2008), pp. 863-892. MR2393431(2009b:37013)

[15] D. Huang, Flow equivalence of reducible shifts of finite type, Ergodic Theory Dynam. Systems 14(1994), pp. 695-720. MR1304139 (95k:46110)

[16] D. Huang, Flow equivalence of reducible shifts of finite type and Cuntz-Krieger algebras, J. reine angew. Math. 462(1995), pp. 185-217. MR.1329907 (96m:46123) 
[17] B. P. Kitchens, Symbolic dynamics, Springer-Verlag, Berlin, Heidelberg and New York (1998). MR $1484730(98 \mathrm{k}: 58079)$

[18] D. Lind and B. Marcus, An introduction to symbolic dynamics and coding, Cambridge University Press, Cambridge (1995). MR1369092 (97a:58050)

[19] K. Matsumoto, On automorphisms of $C^{*}$-algebras associated with subshifts, J. Operator Theory 44(2000), pp. 91-112. MR1774695 (2001g:46147)

[20] K. Matsumoto, Orbit equivalence in $C^{*}$-algebras defined by actions of symbolic dynamical systems, Contemporary Math. 503, Amer. Math. Soc., Providence, RI (2009), pp. 121-140. MR2590619

[21] K. Matsumoto, Orbit equivalence of topological Markov shifts and Cuntz-Krieger algebras, Pacific J. Math. 246(2010), 199-225. MR2645883 (2011k:46086)

[22] K. Matsumoto, Some remarks on orbit equivalence of topological Markov shifts and CuntzKrieger algebras, to appear in Yokohama Math. J.

[23] H. Matui, Homology and topological full groups of étale groupoids on totally disconnected spaces, Proc. London Math. Soc. (3) 104 (2012), pp. 27-56. MR2876963

[24] W. Parry and D. Sullivan, A topological invariant for flows on one-dimensional spaces, Topology 14(1975), pp. 297-299. MR0405385(53:9179)

[25] A. L. T. Paterson, Groupoids, inverse semigroups, and their operator algebras, Progress in Mathematics 170, Birkhäuser, Boston, Basel, Berlin (1999). MR1724106 (2001a:22003)

[26] I. F. Putnam, The $C^{*}$-algebras associated with minimal homeomorphisms of the Cantor set, Pacific J. Math. 136(1989), pp. 329-353. MR978619(90a:46184)

[27] J. Renault, A groupoid approach to $C^{*}$-algebras, Lecture Notes in Math. 793, Springer-Verlag, Berlin, Heidelberg and New York (1980). MR.584266 (82h:46075)

[28] M. Rørdam, Classification of Cuntz-Krieger algebras, K-theory 9(1995), pp. 31-58. MR 1340839 (96k:46103)

[29] J. Tomiyama, Topological full groups and structure of normalizers in transformation group $C^{*}$-algebras, Pacific J. Math. 173(1996), pp. 571-583. MR.1394406 (97i:46124)

[30] J. Tomiyama, Representations of topological dynamical systems and $C^{*}$-algebras, Contemporary Math. 228, Amer. Math. Soc., Providence, RI (1998), pp. 351-364. MR1667670 (99m:46158)

Department of Mathematics, Joetsu University of Education, Joetsu, 943-8512, Japan

E-mail address: kengo@juen.ac.jp 